\title{
A Novel Two-Section Tunable Discrete Mode Fabry-Pérot Laser Exhibiting Nanosecond Wavelength Switching
}

\author{
Richard Phelan, Wei-Hua Guo, Qiaoyin Lu, Diarmuid Byrne, Brendan Roycroft, Paul Lambkin, Brian Corbett, \\ Frank Smyth, Liam P. Barry, Brian Kelly, James O'Gorman, and John F. Donegan
}

\begin{abstract}
A novel widely tunable laser diode is proposed and demonstrated. Mode selection occurs by etching perturbing slots into the laser ridge. A two-section device is realized with different slot patterns in each section allowing Vernier tuning. The laser operates at $1.3 \mu \mathrm{m}$ and achieves a maximum output power of $10 \mathrm{~mW}$. A discontinuous tuning range of $30 \mathrm{~nm}$ was achieved with a side mode suppression greater than $30 \mathrm{~dB}$. Wavelength switching times of approximately $1.5 \mathrm{~ns}$ between a number of wavelength channels separated by $7 \mathrm{~nm}$ have been demonstrated.
\end{abstract}

Index Terms-Fast wavelength switching, semiconductor laser diode, Vernier effect, widely tunable laser.

\section{INTRODUCTION}

W IDELY TUNABLE (WT) monolithically integrated laser diodes have been the subject of considerable interest with the advent of wavelength division multiplexing (WDM). Tunable lasers are needed to reduce the inventory costs associated with different part numbers for fixed wavelength distributed feedback lasers but also to provide new applications such as optical switching and routing which require fast wavelength switching lasers in the nanosecond regime [1], [2]. They are also being used for niche but increasingly important applications such as trace gas detection for environmental emission monitoring and process control [3]. The tuning range of conventional single frequency lasers is approximately $2-5 \mathrm{~nm}$ which significantly under-utilizes the available gain bandwidth of multiple quantum well semiconductor lasers (more than $100 \mathrm{~nm}$ ) and erbium doped fiber amplifiers (about $40 \mathrm{~nm}$ in the $C$ - or $L$-band). Consequently, research efforts have targeted the development of integrated lasers with extended tuning ranges beyond the refractive index limit, an overview can be found in [4]. Typical examples of commercially available monolithic

Manuscript received March 6, 2007; revised November 1, 2007.

R. Phelan, W.-H. Guo, Q. Lu, and J. F. Donegan are with the Semiconductor Photonics Group, School of Physics and Centre for Telecommunication Value-Chain Driven Research (CTVR), Trinity College, Dublin 2, Ireland (e-mail: rphelan@tcd.ie; jdonegan@tcd.ie).

B. Roycroft, P. Lambkin and B. Corbett are with the Photonics Group and Centre for Telecommunication Value-Chain Driven Research (CTVR), Tyndall National Institute, Lee Maltings, Cork, Ireland (e-mail: Brendan.roycroft@tyndall.ie).

F. Smyth and L. P. Barry are with the Research Institute for Networks and Communications Engineering, Dublin City University, Glasnevin, Dublin 9, Ireland (e-mail: liam.barry@dcu.ie).

B. Kelly and J. O'Gorman are with Eblana Photonics Ltd, Unit 32, Trinity Enterprise Centre, Dublin 2, Ireland (e-mail: james@eblanaphotonics.com).

Digital Object Identifier 10.1109/JQE.2007.914219 tuneable laser devices are the sampled grating distributed Bragg reflector (SG-DBR) [5], modulated grating Y-branch (MGY) laser [6], and the digital supermode DBR laser (DS-DBR) [7]. These devices can achieve tuning ranges in excess of $40 \mathrm{~nm}$, SMSRs exceeding $35 \mathrm{~dB}$, output powers above $10 \mathrm{dBm}$, and switching times on the order of $5 \mathrm{~ns}$ using advanced electronic drive circuitry [8]. However, the specific structure of these devices requires complex re-growth and processing techniques, which limit the wafer yield and affect their deployment in large quantities.

Methods for improving spectral purity in edge emitting semiconductor lasers often involve a spatially varying refractive index in the form of a grating. One technique introduced by our group to improve spectral purity in edge-emitting lasers involves the creation of additional features (slots) into the ridge waveguide of a Fabry-Pérot (FP) laser when the ridge itself is formed [9]. The facet reflections in this case are the primary source of feedback necessary for lasing, whilst the slot pattern along the cavity provides additional filtering and hence improved spectral purity. By carefully optimizing the slot position and slot number, lasing with a SMSR of $50 \mathrm{~dB}$ has been achieved. The great advantage of this technique is that it requires no additional re-growth or processing steps. It, therefore, minimizes the fabrication complexity thereby increasing reliability while reducing cost. In this paper, we present results for a novel two-section widely tunable laser diode, which is based on the discrete mode (DM), FP laser structure. The theory behind the use of etched slots has been described previously [10], [11]. The key parameter is the positioning of the slots along the cavity to optimize the optical feedback they produce. By etching slots into the ridge of a waveguide to a controlled depth and location above the active material, an extended tuning range greater than $30 \mathrm{~nm}$ can be achieved. Similar to other tunable diode lasers, the slotted laser uses the Vernier effect to extend the tuning range associated with limited refractive index change. The Vernier effect is a well known technique [12]-[14] for extending the tuning range of widely tunable lasers containing two reflectors with a different grating period, causing a slightly different peak spacing in the reflection spectrum. Lasing occurs when peaks of both sections overlap, so by slightly changing the refractive index of one section two other peaks that are further away will overlap and tuning over a wide wavelength range is obtained.

The slot separation of $35.7 \mu \mathrm{m}$ in the front section and $37.7 \mu \mathrm{m}$ in the back section results in a free spectral range of approx $7 \mathrm{~nm}$ which determines the super-modes spacing and 
enables operation of our device at four discrete super-modes spaced by $7 \mathrm{~nm}$ across the gain spectrum. Fast wavelength switching between these super-modes of approximately $1.5 \mathrm{~ns}$ is demonstrated.

\section{OPERATION PRINCIPLE}

It has been demonstrated that suitable positioning of index perturbations (slots) distributed along the laser waveguide allows the mirror loss spectrum of an FP laser to be manipulated in order to achieve single mode emission [10]. The positioning of the slots is important in maximising the optical feedback they produce, the distance $d$ between the slot features should ideally be

$$
d=\frac{n \lambda}{4 n_{\mathrm{eff}}} \quad n=2,4,6 \ldots
$$

where $\lambda$ is the free space wavelength $n$ is an integer and $n_{\text {eff }}$ is the effective index of the guided mode. In such a scenario the optical feedback will be maximised since reflections from the slots will be in phase. To date DM lasers are designed to operate in a single mode over a wide temperature range. More specifically, for a simple pattern design (1), is applied to calculate the slot spacing $d$, the value of $\lambda$ determines one of the wavelengths where the laser's mirror loss will be reduced due to feedback from the slot pattern. The value of the integer $n$ determines the wavelength separation between adjacent minima in the laser's mirror loss spectra. This wavelength separation $\Delta \lambda$ in the mirror loss spectra is called the effective free spectral range (FSR)

$$
\Delta \lambda=\frac{\lambda^{2}}{2 n_{g} d}
$$

where $n_{g}$ is the group refractive index.

In the case of a two section tuneable laser employing the Vernier effect we require the FSR to be different in each section and also narrow $(<7 \mathrm{~nm})$ so as to produce a comb of mirror loss minima. By increasing the value of $n$ in (1), the slot spacing $d$ increases resulting in a reduction in the FSR in the mirror loss spectrum. By differential tuning of each section a new set of mirror loss peaks can be brought into alignment allowing the lasing wavelength to be tuned. Taking advantage of the Vernier effect tuning mechanism allows the laser to be tuned over a range of $M\left(\Delta n / n_{\text {eff }}\right)$ where $M$ is the number of peaks used and $\Delta n$ is the change in the effective index due to current injection. The maximum number of peaks is given by (3) where $\lambda_{p}$ is the average peak spacing and $\Delta \lambda_{s}$ is the difference in peak spacing between the front and back section

$$
M_{\max }=\frac{\lambda_{P}}{\Delta \lambda_{S}} .
$$

In order to achieve a large tuning range, a large peak spacing $\lambda_{p}$ and a small peak period difference $\Delta \lambda_{s}$ is required. Both of these parameters are already limited by the quasi-continuous tuning range and SMSR. Quasi-continuous tuning is employed to extend the overall wavelength coverage and is achieved

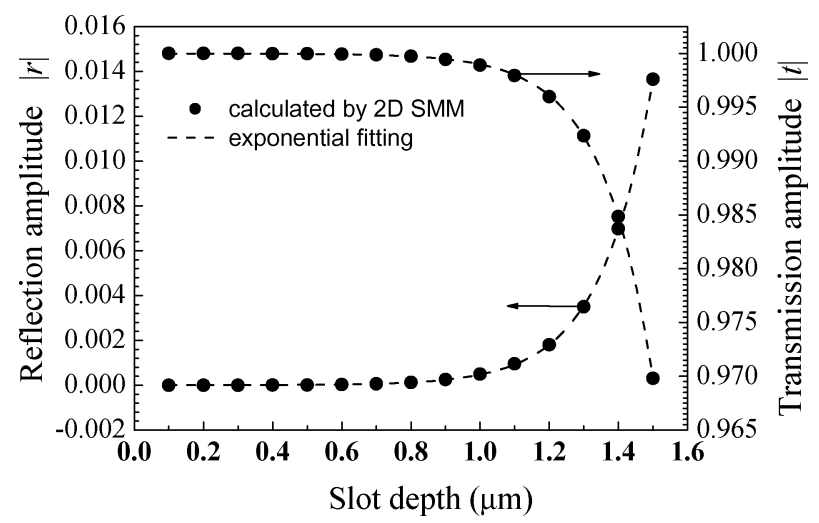

Fig. 1. Reflection and transmission amplitude versus slot depth calculated by the 2-D SMM mode.

by overlapping small regions of continuous tuning from several longitudinal modes. In order to ensure quasi-continuous wavelength coverage over the whole tuning range, the peak spacing $\lambda_{p}$ has to be smaller than the continuous tuning range. In the case of ridge waveguide WT-DM lasers this continuous tuning range is typically around $3 \mathrm{~nm}$. On the other hand, the peak period difference $\Delta \lambda_{s}$ should not be smaller than the width of the reflection peaks, otherwise the SMSR would become impaired. Therefore, a compromise between tuning range and side mode suppression has to be found. The slot pattern chosen in the current design was selected to demonstrate Vernier effect tuning in a two-section DM laser. The peak spacing and peak period difference was approximately 7 and $0.5 \mathrm{~nm}$, which resulted in a large discontinuous tuning range and high SMSR being achieved at the expense of the quasi-continuous tuning range.

For optimization of WT-DM laser diode it is important to know the reflectivity and scattering loss characteristics from structures with multiple slots. We use a 2-D scattering matrix method (SMM), which is described in more detail in [15] to analyze a structure with multiple slots etched into the ridge waveguide. The device structure which was to be fabricated was simulated and as both the guided and radiation modes are taken into account it can predict accurately the loss and reflectivity caused by multiple slots. As illustrated in Fig. 1 it has been shown that the reflection increases while the transmission decreases exponentially as the slot depth is increased so in order to have uniform reflection from multiple slots the slot depth should be precisely controlled. The amplitude reflectivity of a single slot with a slot depth of $1.3 \mu \mathrm{m}$ is calculated to be 0.014 therefore in order to increase the reflectivity and mode selection the number of slots needs to be increased. Fig. 2 plots the reflection and transmission amplitude versus the number of slots calculated with the 2-D SMM model. The reflection amplitude increase and the scattering loss transmission amplitude decreases linearly as the number of slots is increased. Scattering loss also plays a role in spectral selection as the guided modes that are discriminated against by the impressed index of refraction perturbations experience greater scattering loss than the single mode selected for lasing. 


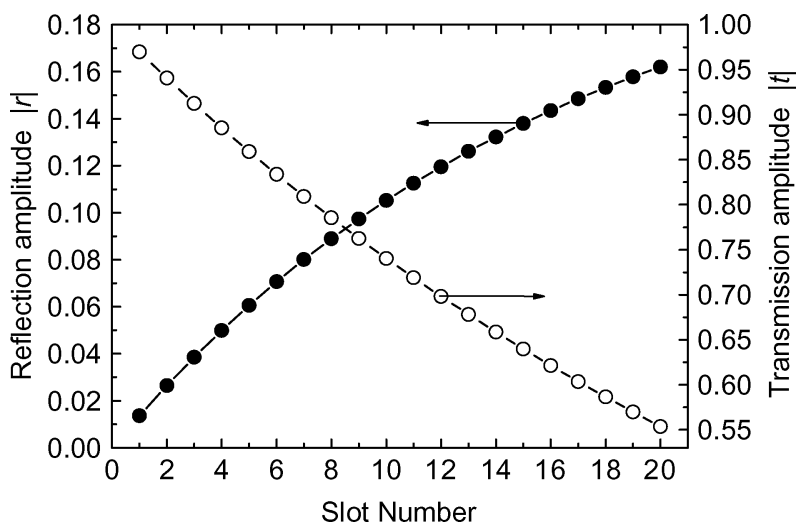

Fig. 2. Reflection and transmission amplitude versus number of slots calculated by the 2-D SMM model.

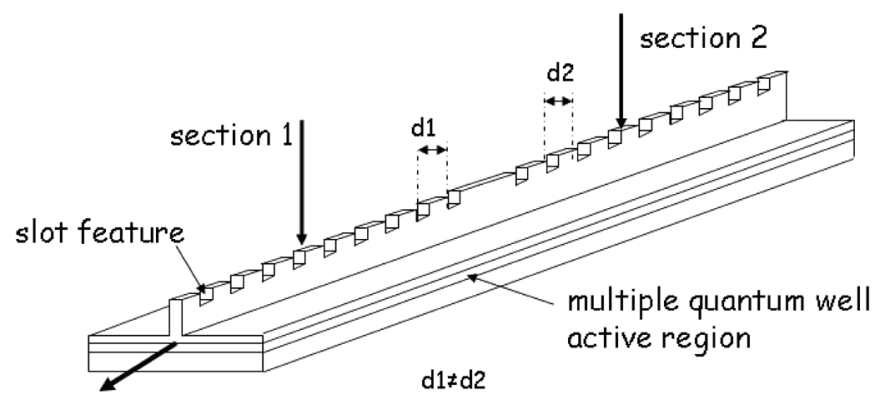

light out

Fig. 3. Schematic of the two-section WT-DM laser.

\section{Device Structure}

A schematic view of the laser is depicted in Fig. 3. It consists of five compressively strained quantum wells, formed from InAlGaAs material with an emission wavelength centered at $1310 \mathrm{~nm}$. The ridge waveguide and slot features were realized using surface processing techniques which do not involve epitaxial regrowth. The cavity is $800 \mu \mathrm{m}$ in length with nine slots etched per section. The ridge width is approximately $2.5 \mu \mathrm{m}$ with a slot width of $1 \mu \mathrm{m}$. A combination of dry and wet etching using etch stop layers was used to define the slot and the ridge depth thus ensuring consistency of device performance. The front and back facets are uncoated with a facet power reflection coefficient of 0.3 .

For Vernier effect tuning, it is necessary that the effective refractive index of each section can be varied independently. To achieve this, the metal and highly doped cap layer was removed in the centre of the ridge to electrically isolate the laser into two sections. The isolation resistance between the two electrodes was measured to be $1 \mathrm{k} \Omega$. The slot period in the front section is $35.7 \mu \mathrm{m}\left(d_{1}\right)$ and the period in the back section is $37.7 \mu \mathrm{m}$ $\left(d_{2}\right)$, this results in a comb-like behavior in the mirror loss spectrum. The comb-like mirror loss spectra of each section possess periodicities of $7 \mathrm{~nm}$ in the front and $6.5 \mathrm{~nm}$ in the back section, which permits the use of Vernier-effect tuning to achieve quasi-continuous wavelength coverage.

Fig. 4(a) shows the calculated reflection spectrum. Lasing will occur at the wavelength where the reflection peaks from

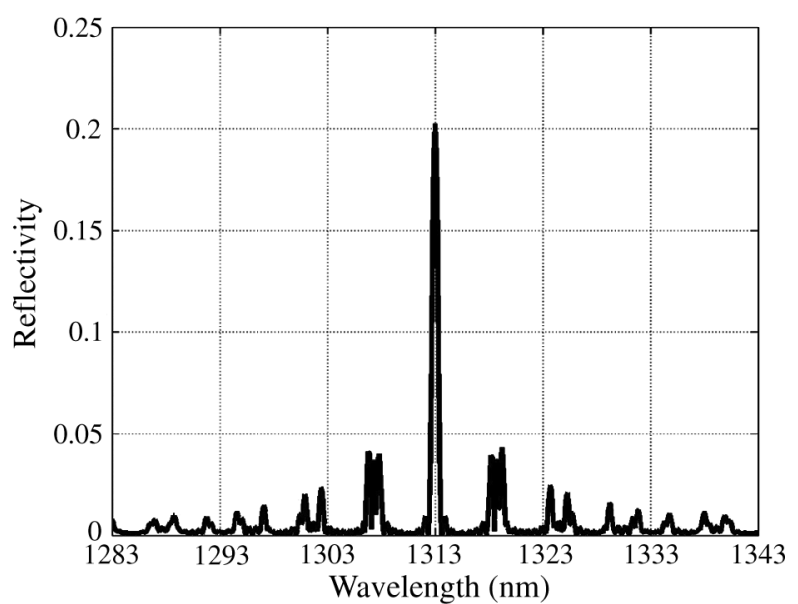

(a)

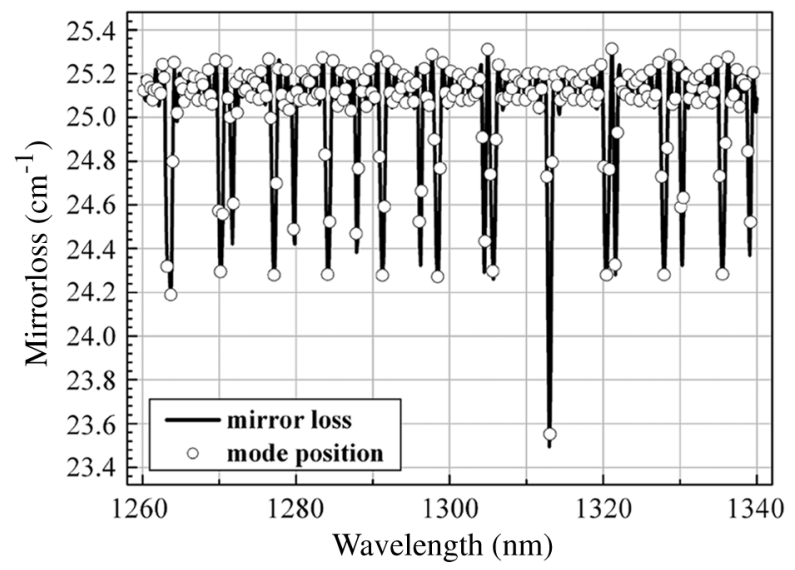

(b)

Fig. 4. Calculated (a) reflectance and (b) mirror loss spectrum and mode position for the two-section device incorporating a different slot pattern in section 1 and 2.

the different sections overlap. Although considering the reflection spectra is helpful in understanding the device principle, it is necessary to investigate the positions of the cavity modes. Hence, Fig. 4(b) shows the calculated mirror loss spectrum for the cavity modes. Lasing will occur at the wavelength where the mirror loss valleys of each section coincide, which is at a wavelength of $1313 \mathrm{~nm}$ in the present example. Large wavelength jumps, so-called super-mode hops, are obtained by varying the current in one section while leaving the other one unchanged whereas continuous tuning is obtained by varying the current in both sections simultaneously.

\section{STATIC CHARACTERIZATION}

The light-current $(L-I)$ and voltage-current $(V-I)$ characteristics of the uncoated $800 \mu \mathrm{m}$ long WT-DM laser are shown in Fig. 5 inset. The $L-I$ curve is linear and the lasing threshold is $28 \mathrm{~mA}$. For the spectral characterization, the light emitted by the diode was collected with a lens-ended single mode fiber, coupled to the front facet of the laser. A fiber-coupled output power of $10 \mathrm{~mW}$ was obtained at a current of $100 \mathrm{~mA}$. Fig. 5 shows superimposed emission spectra from the laser at a heat sink temperature of $20^{\circ} \mathrm{C}$. 


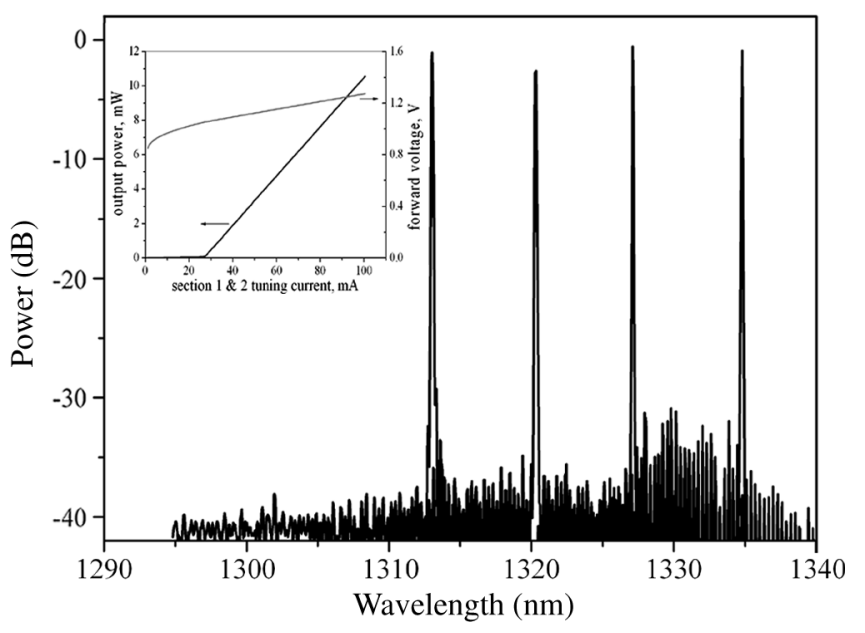

Fig. 5. Superimposed optical emission spectrum of the WT-DM laser measured at a temperature of $20^{\circ} \mathrm{C}$, at various bias currents of section one and two indicating four supermodes. Inset: $L-I, V-I$ characteristics at $25^{\circ} \mathrm{C}$.

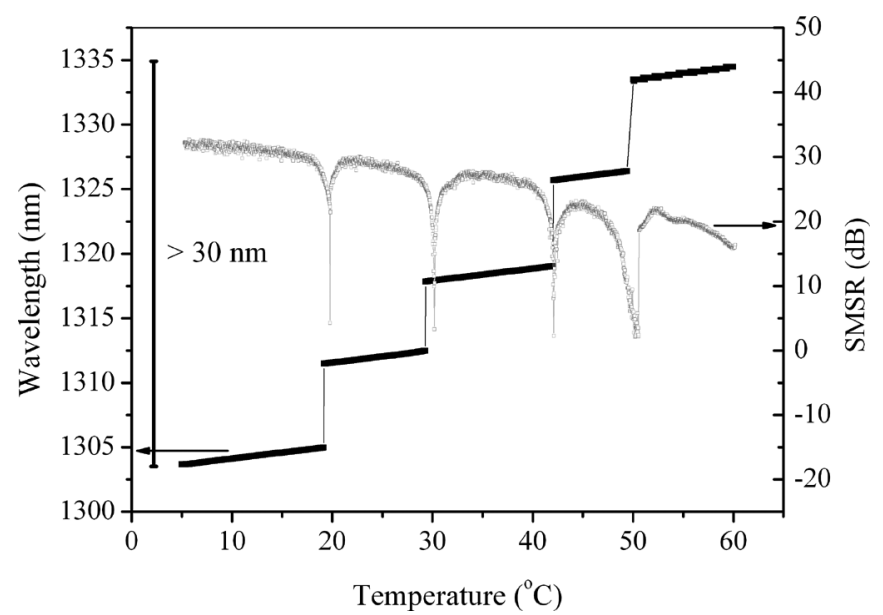

Fig. 6. Emission spectrum and SMSR of the WT-DM laser with section one and two biased at $40 \mathrm{~mA}$ versus heat sink temperature.

The device lases in a single mode with a side mode suppression ratio (SMSR) greater than $30 \mathrm{~dB}$, the emission wavelength is changed by differential tuning of the section one bias current from 20 to $50 \mathrm{~mA}$ while the section two bias current is fixed at $80 \mathrm{~mA}$. Injecting different currents into the two sections of the ridge wave-guide changes the refractive index of the different sections thereby altering the mirror loss spectrum. Within this current range, four super-modes are observed, the spacing of approximately $7 \mathrm{~nm}$ is in good agreement with the mirror loss calculations depicted in Fig. 4.

The laser can be quasi-continuously tuned by varying both tuning currents simultaneously with a maximum continuous tuning range of approximately $1.5 \mathrm{~nm}$ around each supermode. The temperature variation of the laser spectrum was measured with a fixed current of $40 \mathrm{~mA}$ into both sections with the heat sink temperature varied from $5^{\circ} \mathrm{C}$ to $60^{\circ} \mathrm{C}$ as depicted in Fig. 6 . The discontinuous tuning jumps measured are due to new reflection modes coming into alignment and an overall maximum discontinuous tuning range greater than $30 \mathrm{~nm}$ is demonstrated. Within each super mode, the emission wavelength is shifting towards longer wavelength at a rate of $0.1 \mathrm{~nm} /{ }^{\circ} \mathrm{C}$, which is

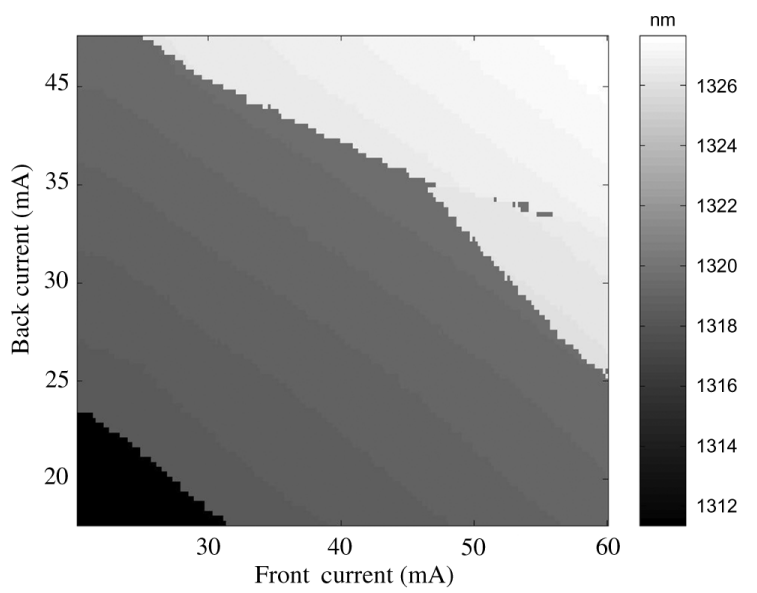

(a)

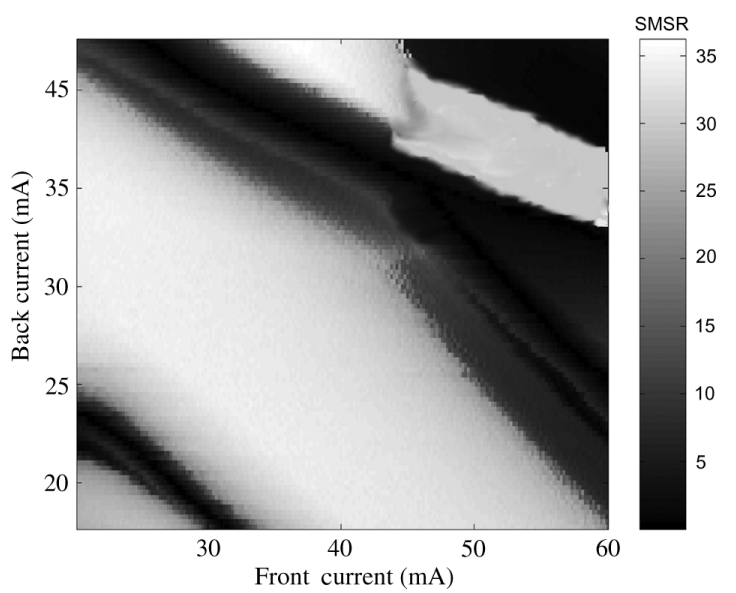

(b)

Fig. 7. (a) Wavelength and (b) SMSR plane as a function of front and back tuning currents.

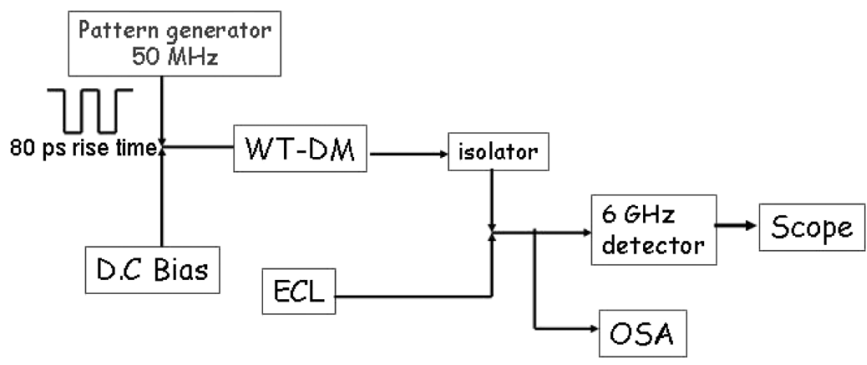

Fig. 8. Optical heterodyne technique used to measure switching speed of WT-DM.

characteristic of thermal tuning. The SMSR falls off at higher temperature due to the temperature dependence of the threshold condition which decreases the power in the lasing mode and hence the SMSR [1].

Fig. 7(a) is the measured wavelength-plane and Fig. 7(b) is the SMSR-plane for the WT-DM as a function of the front and back section tuning currents. On the wavelength surface, several plateaus are apparent, each corresponding to the coincidence of a pair of reflectivity peaks from the slots from section 1 and 2 and termed super-modes. Within these super-modes, the emission wavelength is shifting towards longer wavelength, which is characteristic for the electrothermal tuning. In contrast, by 


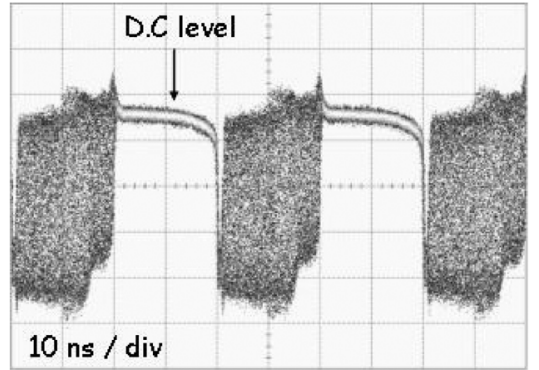

(a)

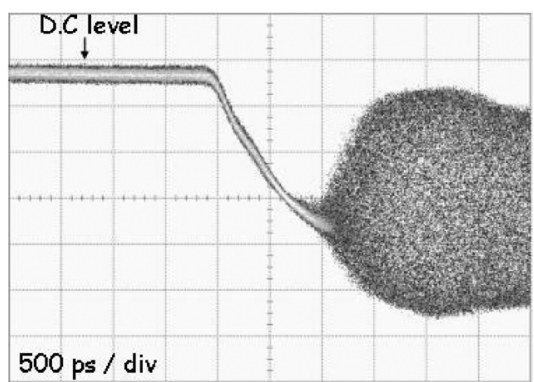

(b)

Fig. 9. Wavelength switching signal of the WT-DM when the laser is switched from channel 1 to channel 2 as viewed on the sampling oscilloscope with (a) 5 ns/div and (b) $500 \mathrm{ps} / \mathrm{div}$.

TABLE I

SWITCHING COMBINATIONS From CHANNEL 1

\begin{tabular}{|l|l|l|l|}
\hline Switch & Channel 2 & Channel 3 & Channel 4 \\
\hline Channel 1 & $1.5 \mathrm{~ns}$ & $1.6 \mathrm{~ns}$ & $1.8 \mathrm{~ns}$ \\
\hline
\end{tabular}

increasing only one tuning current distinct wavelength jumps occur, because another pair of reflection peaks become lined up. Typically, the SMSR is significantly above $30 \mathrm{~dB}$ in regions where the reflection peaks are perfectly lined up as illustrated in Fig. 7(b). The various supermodes are separated by regions with low SMSR, which is the position where another pair of reflections peaks becomes lined up and large wavelength jumps occur.

\section{WAVELENGTH SWITCHING}

Advanced optical networks require fast wavelength switching of tunable lasers in the nanosecond regime. In order to test the switching speed of the two-section laser, an optical heterodyne technique was employed [16] as schematically depicted in Fig. 8. A key requirement for these measurements is a stable, tunable external cavity laser (ECL). The back section of the WT-DM laser was biased at $100 \mathrm{~mA}$ and the front section was driven by a square wave signal at a frequency of $50 \mathrm{MHz}$ generated by a pulse pattern generator with a pulse rise time of $80 \mathrm{ps}$. The square wave changes the current in the front section from 30 to $35 \mathrm{~mA}$, which shifts the emission wavelength of the WT-DM from 1311 to $1318 \mathrm{~nm}$. Here channel 1 is used to denote the super mode at $1311 \mathrm{~nm}$ and channel 2 refers to the super mode at $1318 \mathrm{~nm}$. In this setup, the optical emission from both lasers are mixed into a fibered coupler, its first output is connected to an optical spectrum analyzer (OSA), which allows us to match approximately the ECLs wavelength with channel 2. The ECL is tuned to within $6 \mathrm{GHz}$ of channel 2 to allow the mixing to fall within the bandwidth of the detector. When the WT-DM is switched to channel 2 a heterodyne beat tone between the ECL and channel 2 is generated and can be viewed on an oscilloscope.

A dc signal is generated when the WT-DM is switched to channel 1 as the beat frequency would be in the THz frequency range and fall outside the bandwidth of the detector. Fig. 9(a) illustrates the detected electrical signal as the WT-DM is switched from channel 1 to channel 2 at a repetition rate of
$50 \mathrm{MHz}$. The dc signal corresponds to the time the laser is operating at channel 1 and the heterodyne component corresponds to the time the laser is operating at channel 2. As shown in Fig. 9(b) the laser switches from channel 1 to channel 2 in approximately $1.5 \mathrm{~ns}$. The switching time between channel 1 and several combinations of channels is shown in Table I. A similar switching time can be expected for all channel combinations as the same tuning mechanism is involved in each switch. The wavelength switching time $T_{\mathrm{sw}}$ of the two-section laser can be approximated by [17]

$$
T_{\mathrm{sw}}=\left(\frac{\tau_{c}}{1+\tau_{c} S\left(\frac{d g}{d N}\right)}\right)
$$

where $\tau_{\mathrm{c}}(1 \mathrm{~ns})$ is the stimulated carrier lifetime, $S$ is the photon density in the laser cavity and $d g / d N\left(1 \times 10^{17} \mathrm{~cm}^{2}\right)$ is the differential gain coefficient. Lasers with high values of $S$ and $\mathrm{dg} / \mathrm{dN}$ become good candidates for achieving fast wavelength switching speeds. Using (4) swithching times in the order of $500 \mathrm{ps}$ can be expected for these devices. However, the switching time measured here is limited by the speed of the electrical connections used to bias the laser sections. Although the square wave drive signal has a rise time of $80 \mathrm{ps}$, due to the limited electrical bandwidth of the cables and connections used the rise time is around $1 \mathrm{~ns}$ when it arrives at the laser. Using high speed electrical connector and cables switching times in the order of several hundreds of picoseconds can be expected.

\section{DISCUSSION}

To compare the relative merits of the WT-DM lasers with the current state of the art in tunable lasers which have achieved great success, our results show that initial devices fabricated can discretely tune over a 30-nm range and achieve an SMSR of greater that $30 \mathrm{~dB}$ for these wavelength channels. The overall quasi-continuous tuning range is substantially smaller than that of other Vernier effect tuneable lasers. Significant improvement can be expected by optimal design of the slot configuration and 
by the inclusion of phase tuning sections. It should be noted that unlike the SG-DBR laser that have passive tuning sections, the two-section WT-DM lasers have active sections. This ensures that the carrier lifetimes that affect the switching speed are limited by stimulated carrier lifetimes, and not spontaneous lifetimes as with the SG-DBR, the fastest switching times reported for these lasers are in the order of 5 ns using advanced electronic drive circuitry [8]. It is, thus, possible to achieve very fast switching times in the order of hundreds of picoseconds with these devices. In addition to the performance of these lasers it is also important to emphasise their manufacturability, especially that they can be manufactured using standard optical lithography and surface processing techniques. As a result these devices with optimum design of the slot configuration can be expected to combine the characteristics of SG-DBR devices with the reliability of ridge waveguide laser diodes.

\section{CONCLUSION}

In conclusion we have presented a novel design of a wavelength tunable laser based on a two section slotted FP structure. These devices are easier to fabricate than traditional electronically tunable devices as they are based on a single epitaxial growth step. The tuning characteristics of these widely tunable lasers show distinct regions of high SMSR $>30 \mathrm{~dB}$, where continuous wavelength tuning can be carried out. A discontinuous tuning range of $30 \mathrm{~nm}$ was achieved in these first devices with a super mode spacing of $7 \mathrm{~nm}$, in very good agreement with theoretical predictions. Hence, these observations clearly demonstrate the Vernier effect tuning for the first time in a WT-DM laser diode. A fast wavelength switching time of $1.5 \mathrm{~ns}$ has been experimentally achieved. This two-section device can access four-wavelength channels spaced $7 \mathrm{~nm}$ apart. In the future we plan to realize devices with phase tuning sections working at $1550 \mathrm{~nm}$ with channel spacing as small as $400 \mathrm{GHz}(3.2 \mathrm{~nm})$. To achieve this goal, the slot spacing needs to be increased as well as the number of slots. A larger number of slots will result in a smaller bandwidth of the reflection combs, which will help to preserve the high SMSR. Also because of the smaller channel spacing it will be possible to achieve switching between more channels using the same tuning capability.

\section{REFERENCES}

[1] J. Buus and E. J. Murphy, "Tunable lasers in optical networks," $J$. Lightw. Technol., vol. 24, no. 1, pp. 5-11, Jan. 2006.

[2] L. A. Coldren, "Monolithic tunable diode lasers," IEEE J. Sel. Topics Quantum Electron, vol. 6, no. 11, pp. 988-999, Nov. 2000.

[3] R. Phelan, M. Lynch, J. F. Donegan, and V. Weldon, "Simultaneous multi-species gas sensing using a sampled grating-DBR and modulated-grating Y laser diode," Appl. Opt., vol. 44, pp. 5824-5831, Sep. 2005.

[4] M. C. Amann and J. Buus, Tunable Laser Diodes. Norwood: Artech House, 1998.

[5] V. Jayaraman, Z.-M. Chuang, and L. A. Coldren, "Theory, design, and performance of extended tuning range semiconductor lasers with sampled gratings," IEEE J. Quantum Electron, vol. 29, no. 6, pp. 1824-1834, Jun. 1993.

[6] J.-O. Wesström, G. Sarlet, S. Hammerfeldt, L. Lundqvist, P. Szabo, and P.-J. Rigole, "State-of-the-art performance of widely tunable modulated grating Y-branch lasers," presented at the OFC 2004, Los Angeles, CA, Feb. 2004, Paper TuE2.
[7] A. J. Ward, D. J. Robbins, G. Busico, N. D. Whitbread, P. J. Williams, D. C. J. Reid, and J. R. Rawsthorne, "Modelling of phase-grating based wideband tuneable lasers with simplified quasi-digital wavelength selection," Proc. IEE Optoelectronics, vol. 150, pp. 199-204, Apr. 2003.

[8] J. E. Simsarian, A. Bhardwaj, J. Gripp, K. Sherman, Y. Su, C. Webb, L. Zhang, and M. Zirngibl, "Fast switching characteristics of a widely tunable laser transmitter," IEEE Photon. Technol. Lett., vol. 15, no. 8, pp. 1038-1040, Aug. 2003.

[9] B. Corbett and D. McDonald, "Single longitudinal mode ridge waveguide $1.3 \mu \mathrm{m}$ Fabry-Perot laser by modal perturbation," Electron. Lett., vol. 31, pp. 2181-2182, Dec. 1996.

[10] J. Patchell, D. Jones, B. Kelly, and J. O'Gorman, "Specifying the wavelength and temperature tuning range of a Fabry-Perot laser containing refractive index perturbations," in Proc. SPIE 5825, Jun. 2005, pp. $1-13$.

[11] S. O'Brien and E. P. O'Reilly, "Theory of improved spectral purity in index patterned Fabry-Perot lasers," Appl. Phys. Lett., vol. 86, pp. 201-203, May 2005.

[12] A. Bergonzo, J. Jacquet, D. De Gaudemaris, J. Landreau, A. Plais, A. Vuong, H. Sillard, T. Fillion, O. Durand, H. Krol, A. Accard, and I. Riant, "Widely Vernier tunable external cavity laser including a sampled fiber Bragg grating with digital wavelength selection," IEEE Photon. Technol. Lett., vol. 15, no. 8, pp. 1144-1146, Aug. 2003.

[13] R. Todt, T. Jacke, R. Meyer, J. Adler, R. Laroy, G. Morthier, and M.-C. Amann, "Sampled grating tunable twin-guide laser diodes with Over 40-nm electronic tuning range," IEEE Photon. Technol. Lett., vol. 17, no. 12 , pp. 2514-2516, Dec. 2005.

[14] J. W. Chen, D. N. Wang, and Z. N. Li, "Generation of wavelength-tunable optical short pulses with a highly improved side-mode-suppression ratio over a large wavelength-tuning range," Microw. Opt. Technol. Lett., vol. 46, no. 6, pp. 395-399, Jun. 2005.

[15] Q. Y. Lu, W. H. Guo, R. Phelan, D. Byrne, J. F. Donegan, P. Lambkin, and B. Corbett, "Analysis of slot characteristics in slotted single-mode semiconductor lasers using the 2-D scattering matrix method," IEEE Photon. Technol. Lett., vol. 18, no. 23, pp. 2605-2607, Dec. 2006.

[16] F. Smyth, B. Roycroft, B. Corbett, P. Lambkin, and L. P. Barry, "Fast wavelength switching lasers using two-section slotted Fabry-Pérot structures," IEEE Photon. Technol. Lett., vol. 18, no. 20, pp. 2105-2107, Oct. 2006.

[17] S. Kuwano, Y. Tada, and N. Shibata, "100 ps frequency switching without bit loss for a $10 \mathrm{~Gb} / \mathrm{s}$ ASK modulated signal," IEEE Photon. Technol. Lett., vol. 5, no. 3, pp. 354-356, Mar. 1993.

Richard Phelan received the B.Sc. degree in applied physics with lasers and photonics from the University of Hull, Hull, U.K., in 2000 and the Ph.D. degree in applied physics from Trinity College Dublin, Ireland, in 2004.

He is currently a Postdoctoral Fellow at Trinity College Dublin. His research interests include design and fabrication of novel widely tunable laser diodes for future telecom networks and sensing applications.

Wei-Hua Guo was born in Hubei Province, China, in 1976. He received the B.Sc. degree in physics from Nanjing University, Nanjing, China, in 1998, and the Ph.D. degree from the Institute of Semiconductors, Chinese Academy of Sciences, Beijing, China, in 2004. His doctoral research was on FDTD simulation and fabrication of optical microcavities and design of semiconductor optical amplifiers.

Since September 2004, he has been a Postdoctoral Researcher in the Department of Physics, Trinity College Dublin, Ireland. His current research is focused on optimizing microcavity two-photon absorption photodetectors based on GaAs material system and designing tunable integrated sources for coherent WDM systems.

Qiaoyin Lu was born in Jiangsu Province, China, in 1974. She received the B.Sc. and M.Sc. degrees in optics from the Changchun Institute of Optics and Fine Mechanics, Changchun, China, in 1998 and 2001, respectively, and the $\mathrm{Ph} . \mathrm{D}$. degree at the Institute of Semiconductors, Chinese Academy of Sciences, Beijing, China, in 2004, studying the FDTD simulation and fabrication of photonic microcavities.

She is currently a postdoctoral researcher with the School of Physics, Trinity College Dublin, Ireland. 
Diarmuid Byrne was born in Donegal, Ireland, in 1983. He received the B.A. mod. degree from the School of Physics, Trinity College Dublin, Ireland, in 2005, where he is currently pursuing the Ph.D. degree in physics.

His current research interests include design, characterization and applications of widely tunable semiconductor lasers.

Brendan Roycroft received the B.A.I. degree in microelectronic and electrical engineering and the B.A. in mathematics, and the Ph.D. degree on high-speed response times and optical properties of microcavities from Trinity College Dublin, Ireland, in 1993 and 1998, respectively.

He then worked on twin ridge lasers in the Optoelectronic Research Centre, Tampere, Finland and in the Universidad Carlos III de Madrid, Spain. Since September 2001, he has worked in the Tyndall National Institute, Ireland, and has interests in green and red resonant cavity LEDs, red and near infra-red VCSELs, and novel laser structures and materials. Currently, he is working on the Centre for Telecoms Value-chain-driven Research (CTVR) project, a nationally funded project between Lucent Technologies/Bell Laboratories and all the major Irish universities and is developing integrated tunable lasers for dense wavelength division multiplexing.

Paul Lambkin (M'99) graduated from Loughborough University of Technology, Loughborough, U.K., in 1986 and the Ph.D. degree from the University of Bath, Bath, U.K., in 1990 having worked on nonlinear semiconductor optical waveguides under the supervision of Prof. A. Shore and Prof. M. Adams.

In 1994, he moved to the Physics Department, University College Cork, Cork, Ireland, where he was responsible for the Higher Diploma in Applied Physics. In 1996, he joined the Silicon Technology group in the National Microelectronics Research Centre, University College Cork, where he took responsibility for a micro electromechanical (MEMS) project on microbolometer imaging arrays. Returning to optoelectronics, he joined the Photonics group of the Tyndall National Institute, Cork, Ireland, where he is currently involved in the design of novel semiconductor lasers and the coordination of the European Union and nationally funded research projects.

Dr. Lambkin is a Member of the Institute of Physics and the Optical Society of America.

Brian Corbett received degrees in experimental physics and mathematics and the M.Sc. degree from Trinity College Dublin, Ireland.

He is a Senior Research Scientist and Head of Active Optoelectronic Devices, Photonics Group, Tyndall National Institute, Cork, Ireland. He has 12 years experience in design and fabrication of III-V electronic and optical devices. He has developed extensive III-V micromachining expertise using dry and wet etching techniques. This technology has been applied in both InP- and GaAs-based layer structures for devices such as low-cost single-frequency lasers as well as novel devices such as resonant cavity LEDs and photodetectors. His current main research interest is in the use of geometry to define the optical properties of devices.

Frank Smyth received the B.Eng and M.Eng degrees in electronic engineering from Dublin City University, Dublin, Ireland, in 2001 and 2003, respectively, where he is currently pursuing the Ph.D. in optical telecommunication at the Research Institute for Networks and Communications Engineering (RINCE).

His main topics of research include optical packet switching and fast tunable lasers.
Liam P. Barry received the B.E. degree in electrical engineering and the M.Eng.Sc. degree in optical communications from University College Dublin, Ireland, in 1991 and 1993, respectively.

From February 1993 to January 1996, he was employed as a Research Engineer in the Optical Systems Department of France Telecom's Research Laboratories (CNET), Lannion, France. During this period, his research involved the use of ultrashort optical pulses in high capacity optical networks, and as a result of this work he obtained his Ph.D. degree from the University of Rennes, Rennes, France. In March 1998, he took up a lecturing position in the School of Electronic Engineering, Dublin City University, Ireland, and established the Radio and Optical Communications Laboratory, which is part of the Research Institute for Networks and Communications Engineering (RINCE). He is currently Associate Professor in the School of Electronic Engineering, Director of RINCE, and a Principal Investigator for Science Foundation Ireland. His main research interests involve the development and testing of novel photonic devices and sub-systems for use in next generation optical networks.

Brian Kelly received the Ph.D. degree in physics from Trinity College Dublin, Ireland, in 1996 where he researched the design and optimization of high performance quantum well semiconductor optical switches and their use in image enhancement systems.

Subsequently, he joined Mitsubishi Chemical Corporation, Tokyo, Japan, as a Research Scientist where he worked on the development of high power 980-nm pump lasers for undersea optical amplifier products. He joined Eblana, Dublin, Ireland, in June 2001 and is responsible for laser implementation.

James O'Gorman received the Ph.D. in laser physics by Trinity College Dublin, Ireland, in 1989.

He founded Eblana Photonics with J. Hegarty then he became Professor of Laser Physics at Trinity College Dublin, where he is currently Provost. Subsequently, he joined Bell Laboratories, the R\&D arm of AT\&T (now Lucent Technologies) where he carried out research on high speed laser diode design and high speed data communications applications using laser diodes and laser diode arrays for access and enterprise applications. He was General Manager in Trinity College of Optronics Ireland-the Irish National programme for commercialisation of Optics in Ireland.

John F. Donegan (M'01) received the B.Sc. and Ph.D. degrees in physics from the National University of Ireland, Galway, Ireland.

He held post-doctoral positions in Lehigh University, Bethlehem, PA, and Max Planck Institute, Stuttgart. He is a Professor of physics in Trinity College Dublin, Ireland. His research interests are in photonic structures including broadly tunable lasers based on etched slots, microcavity two-photon absorption detectors, spherical microcavity structures and photonic molecules. He also studies the interaction of quantum dots with human macrophage cells.

Prof. Donegan is a member of the American Physical Society and a fellow of the Institute of Physics and the Institute of Nanotechnology. 